\title{
Malignant melanoma showing smooth muscle differentiation
}

\author{
S S Banerjee, P W Bishop, C M Nicholson, B P Eyden
}

\begin{abstract}
A unique case of a metastatic nondesmoplastic sarcomatoid malignant melanoma in an axillary lymph node showing smooth muscle differentiation in a 54 year old woman is described. The tumour cells exhibited $\alpha$-smooth muscle actin, HHF-35 and desmin positivity but were negative for $\mathrm{S100}$ protein and HMB45. Ultrastructural examination revealed smooth muscle phenotype and there was no evidence of myofibroblastic differentiation, a feature described previously in desmoplastic melanomas.

( $¥$ Clin Pathol 1996;49:950-951)
\end{abstract}

Keywords: malignant melanoma, smooth muscle differentiation, smooth muscle actin, HHF-35, desmin, electron microscopy.

Malignant melanomas show a wide variety of cytological and architectural changes and hence may mimic carcinomas, lymphomas and sarcomas. ${ }^{1}$ Spindle cell melanomas commonly simulate spindle cell carcinomas, peripheral nerve sheath tumours and smooth muscle neoplasms. Schwannian, fibroblastic and myofibroblastic differentiation has also been reported, particularly in desmoplastic melanomas. ${ }^{2-4}$ Here, we document a very unusual case of metastatic non-desmoplastic melanoma in which part of the tumour exhibited immunohistochemical and ultrastructural features of smooth muscle differentiation.

Department of Pathology, Christie Hospital, Manchester S S Banerjee

B P Eyden

Department of Pathology, Wythenshawe Hospital, Manchester P W Bishop

Department of Pathology, Royal Preston Hospital, Preston

C M Nicholson

Correspondence to: Dr S S Banerjee, Department of Pathology, Christie Hospital NHS Trust, Wilmslow Road, Manchester M20 4BX.

Accepted for publication 12 August 1996

\section{Case report}

A 54 year old woman presented in September 1987 with a cutaneous malignant melanoma in the epigastric area. In April 1988 she developed metastases in a right axillary lymph node and within the subcutaneous tissue of the left upper back. Both of these deposits were excised. No other neoplasm was detected on detailed clinical and radiological examination. In July 1994 the patient developed further metastatic disease in the right inguinal nodes, for which a right groin dissection was carried out. In March 1995 the patient was readmitted for lower abdominal pain. On laparotomy, a large mass of nodes was detected around the iliac vessels from which a biopsy specimen was taken. Treatment with decarbazine and interferon was instituted and the patient was alive with a residual right inguinal mass in April 1996.

\section{Pathological findings}

Histological examination of the original cutaneous lesion in the abdominal skin showed a partially regressed invasive malignant melanoma. Assessment of the precise type and thickness of this lesion was difficult. Immunohistochemically, the tumour cells were positive for $\mathrm{S} 100$ protein (Dako, High Wycombe, UK; 1/3000), NKIC3 (Biogenex, Upton-uponSevern, UK; 1/40) and HMB-45 (Dako; 1/50), and negative for cytokeratin (CAM 5.2, Becton Dickinson, Oxford, UK; $1 / 40$; and AE1/3, Biogenex, 1/200) and muscle markers. The axillary lymph node removed in 1988 measured $4.5 \times 2.5 \times 2.0 \mathrm{~cm}$ and was almost completely replaced by a pleomorphic, amelanotic, non-desmoplastic sarcomatoid tumour composed of fascicles of plump spindle cells, polygonal cells and large numbers of multinucleated tumour giant cells containing pleomorphic nuclei and abundant, deeply eosinophilic fibrillar cytoplasm (fig 1A). Numerous mitoses, including atypical forms, were noted. Immunohistochemically, part of this tumour (area A) exhibited positivity with melanocytic markers S100 protein and NKIC3 but not HMB-45; the remainder (area B) showed strong positivity for $\alpha$-smooth muscle actin (Sigma, Poole, Dorset, UK; 1/1500) (fig 1B), HHF-35 (muscle specific actin; Biogenex; $1 / 40$ ), and desmin (D33 clone; Dako; 1/100) (fig 1C). There was also NKIC3 positivity in area B but stains for myoglobin (Dako; 1/1000), fast myosin (Sigma; 1/600), HMB-45, and $\mathrm{S} 100$ protein were negative.

For ultrastructural examination, tissue from the wax block was deparaffinised in xylene and embedded in epoxy resin. Areas A and B contained cells with moderate amounts of rough endoplasmic reticulum cisternae. Unambiguous melanosomes were not identified. Area B showed modestly developed subsurface tracts of smooth muscle myofilaments with focal densities, which were absent from area A (figs $2 \mathrm{~A}$ and $2 \mathrm{~B}$ ). The fibronexus junctions defining myofibroblastic differentiation were not observed. ${ }^{5}$ The metastatic deposits in the subcutaneous tissue of the back and in the inguinal and intrapelvic lymph nodes exhibited typical features of metastatic melanoma with predominantly large epithelioid cells admixed with a few fascicles of plump spindle cells. The subcutaneous deposit contained moderately abundant melanin pigment. Immunostains revealed S100 protein, NKIC3 and HMB-45 positivity. Stains for muscle markers and cytokeratin were negative.

\section{Discussion}

Malignant melanomas, particularly the desmoplastic and neurotropic variants, may show 


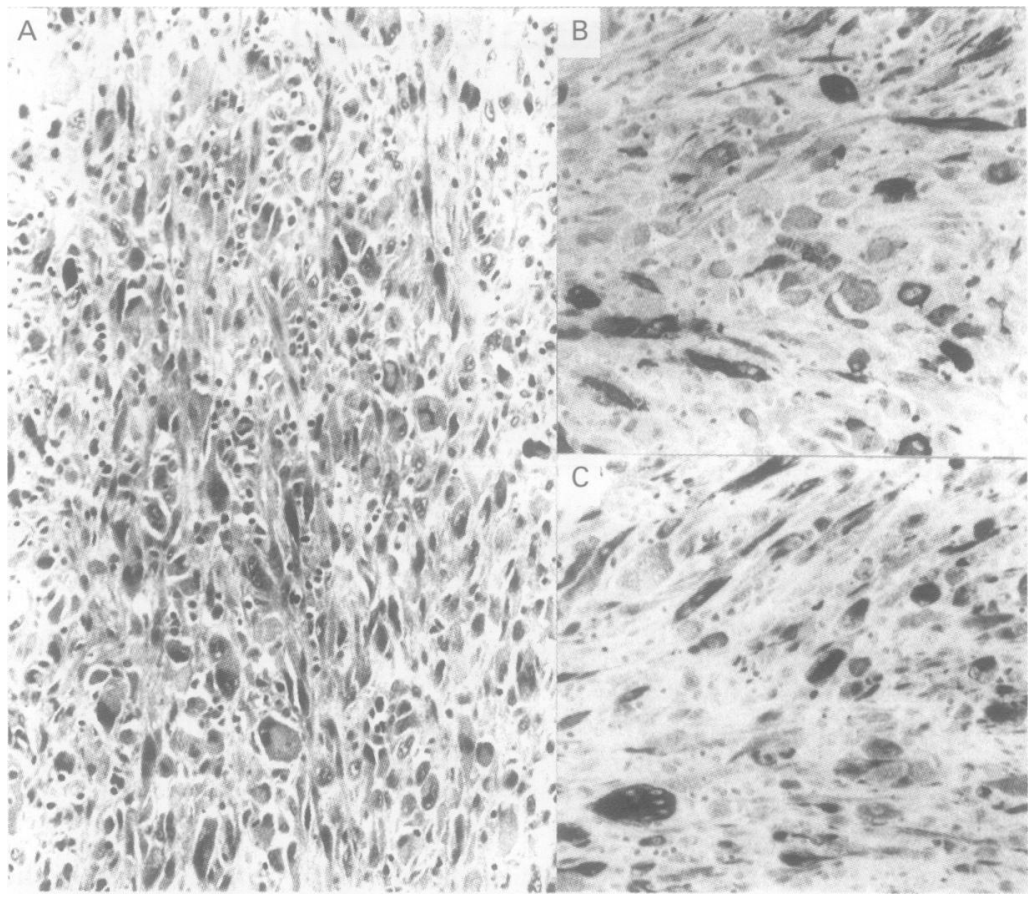

Figure 1 (A) Pleomorphic sarcomatoid area in the axillary node metastasis of the melanoma (haematoxylin and eosin). (B) Strong smooth muscle actin positive tumour cells. (C) Desmin positive tumour cells.

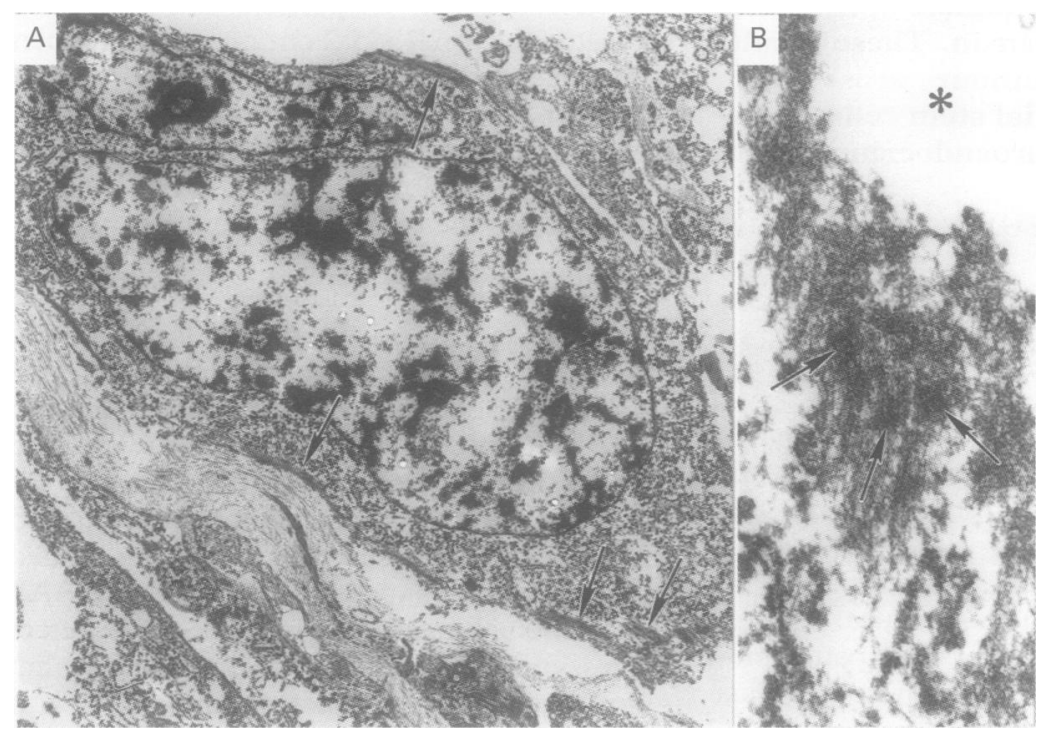

Figure 2 Ultrastructure of tumour cells from the area showing smooth muscle immunophenotype. (A) The cytoplasm contains many rough endoplasmic reticulum cisternae, which are inconspicuous at this magnification, and peripheral bundles of smooth muscle myofilaments (arrows) (original magnification $\times 5000$ ). (B) High power of myofilament bundle showing focal densities (arrows) (original magnification $\times 40000$ ). * Extracellular space. desmin, have been documented in some melanocytic tumours. Bittesini et $a l^{8}$ demonstrated desmin positivity in a metastatic melanoma in which the neoplastic cells showed rhabdoid phenotype as a result of accumula- $\frac{\rho}{5}$ tion of paranuclear whorls of intermediate fila- 0 ments. Truong et al $^{9}$ also demonstrated desmin $\stackrel{0}{*}$ in rare cells in one metastatic melanoma. $\frac{\text { o }}{2}$ Reiman $e t a l^{3}$ found desmin positivity in three $\vec{F}$ neurotropic and four of six usual desmoplastic $\stackrel{5}{+}$ melanomas of the head and neck. Ultrastruc- $\frac{C}{D}$ tural examination of four of these lesions $\frac{\bar{O}}{\bar{c}}$ revealed features, according to these authors, $\frac{\widehat{\Phi}}{\mathrm{D}}$ of fibroblastic and myofibroblastic differentia- $\frac{\Omega}{\nu}$ tion. We have previously identified an ${ }^{\infty}$ $\alpha$-smooth muscle actin positive melanoma, $\vec{O}$ which was negative for HHF-35 and desmin. ${ }^{10} \overrightarrow{\vec{H}}$ We have subsequently encountered two cases $\stackrel{\omega}{\sigma}$ of desmoplastic melanoma showing smooth muscle actin positivity (unpublished observation). Recently, Carlson et $a l^{2}$ also demon- $\stackrel{\oplus}{-}$ strated smooth muscle actin positivity in $12 \vec{~}$ (52\%) of 26 cases of desmoplastic neurotropic 융 melanoma. They interpreted this result as a응 non-specific phenomenon as these tumours did not exhibit any myofibroblastic or smooth $z$ muscle differentiation at the ultrastructural level. We are, however, unaware of any $\frac{\mathbb{}}{3}$ documented case of melanoma showing the full immunophenotypic and ultrastructural spectrum of smooth muscle differentiation. $\mathscr{E}$ The present case exhibited $\alpha$-smooth muscle? actin, HHF-35 and desmin positivity. In addition, fine filaments with focal densities were detected at the ultrastructural level in the cells exhibiting smooth muscle immunophenotype $\frac{\circ}{\mathbb{D}}$ and features of myofibroblastic differentiation $\cong$ were absent. ${ }^{5}$ These cells lost reactivity to $S 100$ 음 protein and HMB-45 but, interestingly, re- 3 tained positivity to NKIC3. It is noteworthy that subsequent metastatic deposits of the tumour did not exhibit any evidence of smooth muscle differentiation and the precise cause of $\overline{3}$ this transient aberration remains uncertain.

We wish to thank Mrs E Ryan for secretarial help.

1 Mooi WJ, Krausz T. Cutaneous melanoma. In: Biopsy pathology of melanocytic disorders. London: Chapman and $\frac{7}{3}$
Hall, 1992:281-9.

2 Carlson JA, Dickersin GR, Sober AJ, Barnhill RL. Desmoplastic neurotropic melanoma: a clinicopathologic analysis $N$ plastic neurotropic melanoma: a clinico

3 Reiman HM, Goellner JR, Woods JE, Mixter RC. N Desmoplastic melanoma of the head and neck. Cancer $N$ 1987;60:2269-74

4 From L, Hanna W, Kahn HJ, Gruss J, Marks A, Baumal R. Origin of desmoplasia in desmoplastic malignant
melanoma. Hum Pathol 1983;4:1072-80.

Schwannian, fibroblastic or myofibroblastic differentiation at the ultrastructural level. ${ }^{2-4}$ Very rarely, osteocartilaginous metaplasia has been noted in melanomas ${ }^{6}$ and there are occasional reports of rhabdomyosarcomatous differentiation in giant congenital naevi. ${ }^{7}$ Schwannian differentiation in melanocytic lesions is not an unexpected phenomenon as both melanocytes and Schwann cells are derived from the neural crest. However, mesenchymal differentiation with formation of heterologous elements in melanocytic tumours is an unusual occurrence. Immunohistochemically positive muscle markers, either smooth muscle actin or
5 Eyden BP. Brief review of fibronexus and its significance for $\mathbb{D}$ myofibroblastic differentiation and tumour diagnosis. $\stackrel{\mathscr{C}}{+}$ Ultrastruct Pathol 1993;17:611-22.

6 Lucas DR, Tazelaar HD, Unni KK, Wold LE, Okada K, Dimarzio DJ, et al. Osteogenic melanoma: a rare variant of malignant melanoma. Am $\Im$ Surg Pathol 1993;17:400-9.

7 Schmitt FC, Bittencourt A, Mendonca N, Dorea M. Rhab-

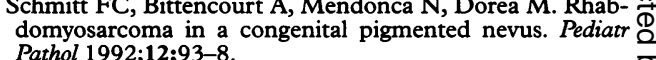

8 Bittesini L, Dei Tos AP, Fletcher CDM. Metastatic malignant melanoma showing a rhabdoid phenotype: $\Omega$ further evidence of a non-specific histological pattern. His-응 topathology 1992;20:167-70

9 Truong LD, Rangdaeng S, Cagle P, Ro JY, Hawkins H, Font RL. The diagnostic utility of desmin: a study of 584 cases and review of the literature. Am $\mathcal{F}$ Clin Pathol 1990;93:305-14.

10 Bishop PW, Menasce LP, Yates AJ, Win NA, Banerjee SS An immunophenotypic survey of malignant melanomas. Histopathology 1993;23:159-66. 